\title{
Manejo anestésico de histerectomía más doble anexectomía por tumor borderline de ovario izquierdo en paciente con síndrome de sensibilidad química múltiple
}

\author{
B. Esnaola Iriarte, S. Irigoyen Miró y F. Torre Mollinedo
}

Servicio de Anestesiología, Reanimación y Tratamiento del Dolor. Hospital de Galdakao-Usansolo, Vizcaya. España

\section{ABSTRACT}

The multiple chemical sensitivity syndrome (MCS), also known as idiopathic environmental intolerance (IAI), among others, is a complex and poorly defined disorder that produces various symptoms in response to various stimuli. There is a lack of valid studies that establish the pathogenesis of this syndrome. The anesthetic management of these patients is a challenge for anesthesiologists, due to the fact that there are no established guidelines. We present a case of successful surgery in a patient that suffers from MCS by performing a conventional TIVA enhanced with a thorough premedication and the latex allergy protocol.

Key words: Multiple chemical sensitivity.

\section{RESUMEN}

El síndrome de sensibilidad química múltiple (SQM), también conocido como intolerancia ambiental idiopática (IAI), entre otros, es un desorden complejo y mal definido que produce diversos síntomas en respuesta a diferentes estímulos. No hay estudios válidos que establezcan la patogénesis de este síndrome. El manejo anestésico de estos pacientes es un reto para los anestesiólogos, dado que no hay unas pautas de actuación establecidas. Se presenta un caso de cirugía exitosa en una paciente afecta de SQM realizándose una anestesia total intravenosa (TIVA) convencional a la que se añadió una premedicación exhaustiva y la aplicación del protocolo de alergia al látex.

Palabras clave: Sensibilidad química múltiple.

que se trata de una enfermedad predominantemente padecida por mujeres.

Entre los síntomas más comunes que padecen los afectados de SQM después de haber presentado una exposición química se encuentran: fatiga extrema, dolor de cabeza, problemas gastrointestinales, mareos, vértigos, ansiedad, depresión, irritación de las vías respiratorias superiores, dolor de músculos y articulaciones, dificultad de memoria y de concentración (2).

El control ambiental es una pieza clave y esencial para el éxito del tratamiento. Este ha de realizarse siempre por personal formado en esta materia y es prescrito tras estudiar las individualidades de cada paciente. 
Desde el punto de vista anestésico, el Institut Ferran de reumatología, pionero en el diagnóstico del síndrome de SQM, ofrece desde el 2006 en su página web un documento de recomendaciones anestésicas y farmacológicas para el manejo de estos pacientes. Fuera de esto, no existen pautas de actuación establecidas en cuanto a la anestesia en estos pacientes.

Las principales recomendaciones anestésicas que se hacen en este documento de consenso son las siguientes (3): premedicar con una benzodiacepina de corta/ media duración, realizar una hidratación con suero fisiológico o Ringer lactato a 15-20 ml/kg, emplear el propofol como hipnótico, el fentanilo como analgésico y evitar los relajantes musculares si es posible. En caso de tener que utilizar relajantes, el de elección sería el vecuronio, y siempre acompañado de monitorización neuromuscular para evitar problemas de sobredosificación. Para la analgesia del postoperatorio inmediato recomiendan analgésicos habituales como paracetamol asociado a un AINE, y en caso de intolerancia debería asociarse un opioide tipo tramadol o morfina a dosis bajas. En caso de realizarse una anestesia regional recomiendan el uso de anestésicos locales tipo amida evitando la adrenalina.

En este marco, presentamos el manejo anestésico de una paciente afecta de SQM que precisó cirugía ginecológica por presentar una tumoración gigante de probable origen anexial.

\section{CASO CLÍNICO}

Se trata de una mujer de 53 años diagnosticada de SQM programada para cirugía ginecológica por una masa abdominal gigante de probable origen anexial, compatible con cistoadenocarcinoma sin aparentes signos de diseminación. El diagnóstico se realizó mediante TAC sin contraste. Como antecedentes médicos describe: sensibilización de contacto a cloruro de mercurio (a raíz de colocación de implantes dentales, lo cual motivó el diagnóstico de SQM), intolerancia al látex y a múltiples fármacos y alimentos, hiperlaxitud de larga evolución con escoliosis, cistitis de repetición de carácter irritativo, espondiloartrosis, disfunción de articulación temporo-mandibular, síndrome de túnel carpiano bilateral y trastorno adaptativo con síntomas ansiosodepresivos. Como antecedentes quirúrgicos destacan: hernioplastia umbilical y esclerosis de hemorroides, intervenidas previamente al diagnóstico de SQM y que cursaron sin incidencias. No refiere tomar tratamiento de forma habitual. La exploración de la vía aérea no mostró datos predictores de vía aérea difícil y se clasificó como ASA II.

La paciente ingresó la misma mañana de la cirugía (ante la ausencia de un protocolo definido en nuestro hospital para actuar en el síndrome SQM se decide seguir las guías del instituto Ferrán] y se aplicó el protocolo hospitalario de alergia al látex. La noche anterior se instauró un aspirador de partículas ambientales en el quirófano asignado a la intervención, el cual se mantuvo durante toda la intervención, y se retiraron todos aquellos utensilios o productos que contuvieran látex. Tras canalizar dos vías periféricas de $18 \mathrm{G}$, se premedicó a la paciente con 2 mg de midazolam,
$5 \mathrm{mg}$ de desclorfeinamina, $50 \mathrm{mg}$ de ranitidina y $4 \mathrm{mg}$ de dexametasona, además de fluidoterapia con suero fisiológico al 0,9\% (20 ml/kg]. Dado que iba a ser una cirugía abierta, se le ofreció la posibilidad de colocación de un catéter epidural para la analgesia postoperatoria, pero la paciente lo desestimó. Acto seguido, se realizó la monitorización de tensión arterial (TA no invasiva), ECG, pulsioximetría, diuresis, BIS y TOF. Tras preoxigenar a la paciente durante 3 minutos con $0_{2}$ al $100 \%$, se procedió a la inducción anestésica administrándose fentanilo i.v. 3-6 $\mu \mathrm{g} / \mathrm{kg}$, propofol en modo TCl a $4 \mathrm{mcg} / \mathrm{ml}$ y rocuronio $0,6 \mathrm{mg} / \mathrm{kg}$. Se intubó a la paciente con un tubo del número 7,5 G, visualizándose un CormackLehane de I/IV a la laringoscopia directa y se conectó a la paciente a ventilación mecánica controlada por volumen. El procedimiento transcurrió sin incidencias. El mantenimiento se realizó también con propofol en modelo TCl a 2,5 mcg/ml, fentanilo $1-2 \mu \mathrm{g} / \mathrm{kg}$ y rocuronio 0,3 $\mathrm{mg} / \mathrm{kg}$. Dada la extensión de la masa anexial se le practicó finalmente una histerectomía con doble anexectomía, apendicectomía y omentectomía con toma de biopsias peritoneales múltiples. Durante las $3 \mathrm{~h}$ de intervención la paciente se mantuvo en todo momento hemodinámicamente estable y normocápnica. Las dosis anestésicas totales fueron $550 \mu \mathrm{g}$ de fentanilo, $90 \mathrm{mg}$ de rocuronio y $990 \mathrm{mg}$ de propofol. Como analgesia previa a la salida del quirófano se le administraron $50 \mathrm{mg}$ de dexketoprofeno junto con $4 \mathrm{mg}$ de ondansetrón y se le practicó un bloqueo transverso abdominal (TAP) bilateral con $40 \mathrm{ml}$ de ropivacaína al $0,375 \%$ (Figura 1). La paciente pudo ser extubada sin incidencias y se trasladó a la Unidad de Recuperación Postanestésica (URPA) las siguientes dos horas, donde se le colocó un infusor elastomérico de morfina i.v. a $1 \mathrm{mg} / \mathrm{h}$ para el dolor agudo postoperatorio. En las próximas horas la paciente fue trasladada a la planta de ginecología, donde se le proporcionó una habitación individual y el aspirador de partículas del quirófano, así como sábanas antialergénicas. Su evolución en la planta fue satisfactoria, por lo que se pudo retirar el infusor de morfina a las 72 h y fue dada de alta sin evidencia de complicaciones a los 4 días.

\section{DISCUSIÓN}

La SQM también denominada como "enfermedad ambiental idiopática" o "alergia universal", entre otros, se describe por primera vez en 1950, cuando se observó cómo algunas personas presentaban una serie de síntomas cuando se exponían a niveles muy bajos de sustancias ambientales, laborales y domésticas. La OMS no ha clasificado todavía la SQM en la Clasificación Internacional de Enfermedades (CIE), pero ya hay algunos países que sí la han incorporado en sus respectivas CIE a nivel nacional. En España, la SQM se encuentra clasificada en el CIE 10 bajo el código T78.40 "Alergia no especificada", lo que claramente la sitúa fuera de las enfermedades de origen psicológico.

Los síntomas informados de exposición química son diversos y variables de un paciente a otro, pero fundamentalmente incluyen: dolor de cabeza, dolor en músculos y articulaciones, confusión, disfunción cognitiva, síntomas asmáticos, rinitis, alteración del sueño, fatiga 

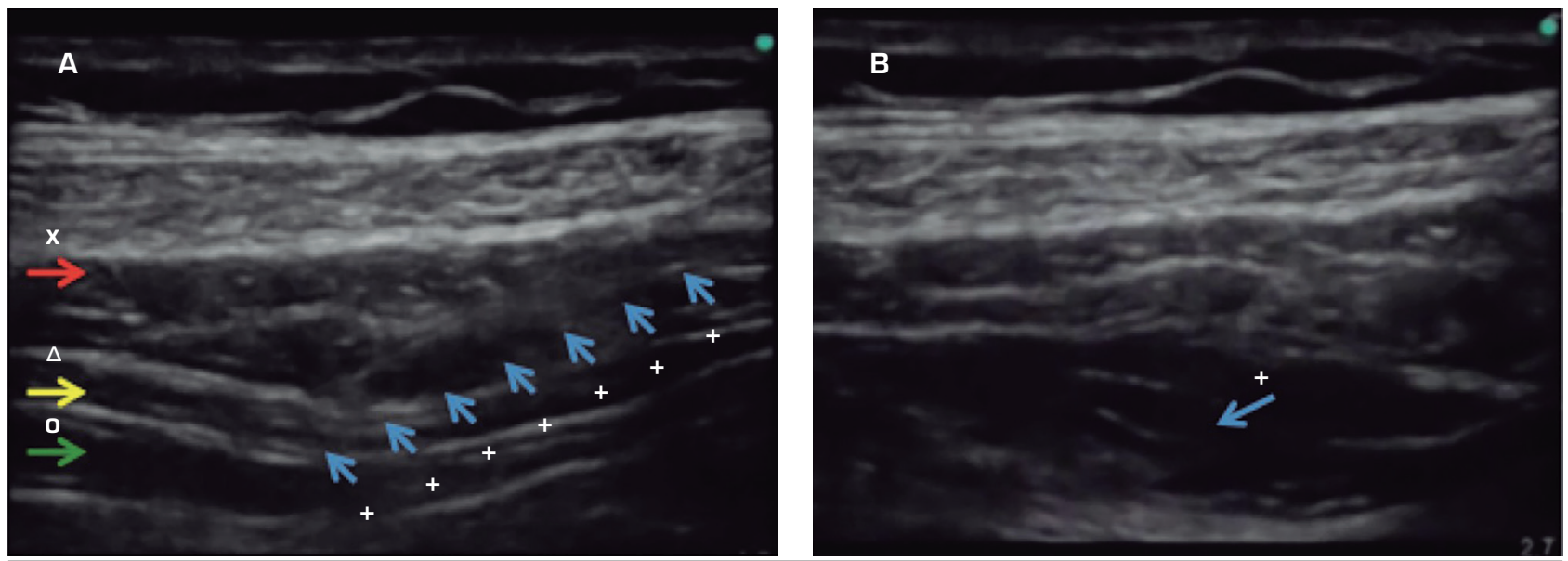

Fig. 1. Bloqueo TAP. A. En sentido craneocaudal se observan las diferentes capas de la musculatura abdominal: músculo oblicuo externo (flecha roja [x]), oblicuo interno (flecha amarilla [ $\Delta$ ]) y transverso del abdomen (flecha verde [o]). El bloqueo TAP se realiza en la fascia situada entre los músculos oblicuo interno y transverso del abdomen. Se observa la aguja insertada en el plano del bloqueo TAP (flechas azules [+]). B. Se observa el anestésico local distribuido por la fascia, desplazando hacia abajo el músculo trasverso del abdomen.

$y$, en algunos casos, síntomas psiquiátricos como ansiedad y depresión, y de forma menos frecuente rabia e ira [2]. En la revisión publicado por Sorg [4], se describieron 41 síntomas distintos, muchos de los cuales los padecían solamente a una minoría de pacientes.

En el momento actual, la SQM es objeto de múltiples estudios. En su etiopatogenia podrían participar factores genéticos, químicos, alergénicos y anatómicos. Podría desencadenarse tras un accidente o experiencias vitales traumáticas, ya que está relacionado con una mayor incidencia de ansiedad y depresión y síndromes como la fibromialgia, el ojo seco y el asma. Dada la ausencia de marcadores específicos, el diagnóstico es fundamentalmente clínico.

No existe un conjunto de criterios diagnósticos definitivos de SQM. Actualmente, los criterios de Cullen y los de la Conferencia de Consenso sobre SQM de 1999 son los más aceptados (5). Cullen (6) define un caso de SQM cuando se cumplen los siguientes criterios:

1. El trastorno se adquiere después de una exposición ambiental, insulto o enfermedad documentada.

2. Los síntomas involucran múltiples órganos y sistemas.

3. Los síntomas se repiten y disminuyen en respuesta a estímulos predecibles.

4. Los síntomas son provocados por exposiciones a sustancias químicas de diversas clases estructurales y modos de acción toxicológicos.

5. Los síntomas son provocados por exposiciones que son demostrables.

6. Los niveles de exposición que provocan síntomas deben ser muy bajos, generalmente inferiores al $1 \%$ de los valores límite de umbral establecido.

7. Ninguna prueba complementaria disponible es capaz de explicar los síntomas.

Hoy en día, la SQM no tiene un tratamiento específico. Existen hospitales que han comenzado a elaborar protocolos de actuación y manejo perioperatorio de estos pacientes. Uno de ellos es el Institut Ferrán de Reumatología (3), que recoge el documento realizado por el Servicio Catalán de la Salud, reconocido como único protocolo sobre síndrome SQM en España, junto con un listado de fármacos a evitar (Tabla I). La contraindicación de dichos fármacos en estos pacientes podría explicarse por diferentes mecanismos (7): sus posibles efectos adversos, como broncoconstricción (neostigmina, atropina o succinilcolina), la liberación de histamina (pentotal, morfina y la mayoría de los relajantes neuromusculares] o su capacidad alergizante [ácido paraaminobenzoico: metabolito inactivo de los anestésicos locales tipo éster].

Ante el caso que se nos presentaba, al revisar la literatura no hallamos apenas información acerca de las pautas de actuación en este tipo de patología, salvo el documento creado por el Servicio Catalán de Salud mencionado previamente. M. T. Fernández y J. C. Álvarez (7), anestesiólogos del Hospital de Medina del Campo de Valladolid, describen un caso de uso de sevoflurano como agente anestésico único para cirugía de senos paranasales en una paciente con SQM, obte-

TABLA I

FÁRMACOS QUE DEBEN EVITARSE

\begin{tabular}{|l|l|}
\hline $\begin{array}{l}\text { Adrenalina y otras } \\
\text { aminas adrenérgicas }\end{array}$ & $\begin{array}{l}\text { Morfina y derivados } \\
\text { [salvo fentanilo] }\end{array}$ \\
\hline Aminofilina & Neostigmina \\
\hline Anestésicos locales & Relajantes musculares \\
\hline Fármacos colinérgicos & succinilicolina \\
\hline $\begin{array}{l}\text { Fármacos } \\
\text { antcolinesterásicos }\end{array}$ & Furosemida \\
\hline
\end{tabular}


Rev. Soc. Esp. del Dolor, Vol. 26, N. ${ }^{\circ}$ 4, Julio-Agosto 2019

niendo buenos resultados. Según relatan los autores, eligen el sevoflurano, tanto en la inducción anestésica como durante el mantenimiento de la misma, para evitar así, gracias a sus propiedades relajantes, el uso de bloqueantes neuromusculares (BNM), como ya se recomienda en algunos artículos (8). Lorenzo y cols. (8), anestesiólogos del Hospital Río Hortega de Valladolid, presentan un caso deł manejo anestésico de una cesárea electiva en una mujer de 34 años diagnosticada de SQM. En este caso se llevó a cabo anestesia subaracnoidea con $12 \mathrm{mg}$ de bupivacaína hiperbara sin observarse ninguna complicación intraoperatoria, salvo un episodio de hipotensión que se resolvió tras la administración i.v. de $30 \mathrm{mg}$ de efedrina.

En nuestro caso, al tratarse de un síndrome de hipersensibilidad, decidimos realizar la premedicación como si se tratara de un caso de alergia al látex o mastocitosis (patologías sobre las cuales existe un protocolo de actuación en nuestro Hospital). Al igual que Fernández y cols., realizamos una hidratación abundante, puesto que en diferentes estudios se ha observado que los pacientes con SQM presentan una respuesta cardiovascular anómala e hipotensión ortostática (9), relacionadas con una disfunción autonómica. Como agente inductor y para el mantenimiento utilizamos el propofol en modo TCl, sin presentar ningún tipo de complicación. El propofol, por sus propiedades farmacocinéticas y farmacodinámicas, parece ser un fármaco seguro para estos pacientes, aunque sería conveniente descartar primero alergia a alguno de sus componentes como la soja o la yema de huevo. A pesar de ser muy baja la incidencia de efectos adversos del tipo inmunológico, hay descrito un caso de broncoespasmo anafiláctico tras infusión de propofol en un paciente con SQM (9). Como bloqueante neuromuscular se decidió utilizar un BNM de tipo aminoéster, como es el rocuronio, dado que la liberación de histamina, y por lo tanto el riesgo de anafilaxia, es menor en comparación con los BNM de tipo benzilisoquinolinas (10). Durante su administración no se detectó ningún tipo de efecto adverso. Como analgésico, utilizamos fentanilo durante la intervención, administrándolo de forma lenta, por ser un opiáceo no liberador de histamina. Como analgesia en el postoperatorio, optamos por un tratamiento multimodal, realizamos un bloqueo TAP bilateral con ropivacaína tras cerrar la incisión y utilizamos morfina i.v. en el postoperatorio inmediato, junto con AINE, sin objetivar ningún tipo de complicación. La paciente pudo ser dada de alta a los 4 días de manera satisfactoria.

\section{CONCLUSIONES}

Hay muy poco escrito sobre la SQM, y la falta de información en la literatura respecto al apropiado manejo anestésico en estos pacientes supone un reto para el anestesista. Como filosofía general, sería recomendable evitar los medicamentos que los pacientes identifican como aquellos a los que han tenido respuestas adversas. Nuestra experiencia con este caso ha sido que es posible abordar un plan anestésico basado en propofol, fentanilo y rocuronio, además de una técnica de anestesia regional con ropivacaína y morfina para el postoperatorio de manera satisfactoria en pacientes con SQM.

\section{BIBLIOGRAFÍA}

1. Puri B, Treasaden I. Psychiatry an Evidence Based Text. Hodder Education; 2011. p. 793-815.

2. Ballantyne B, Marrs T, Syversen T. Toxicological questions and mechanisms. Londres: John Wiley \& Sons; 2009. p. 2303-54.

3. Montes Pérez A. Documento sobre recomendaciones en la anestesia y fármacos a evitar en la I/SQM. Institut Ferran de Reumatología. Disponible en: http://www. institutferran. org/intoleranciaquímica.htm

4. Sorg BA. Multiple Chemical Sensitivity: potential role of neural sensitization. Critical Reviews in Neurobiology. 1999;13(3):283-316.

5. Rossi S, Pitidis A. Multiple chemical sensitivity: review of the state of the art in epidemiology, diagnosis and future perspectives. J Occup Environ Med. 2018;60(2):138-46. DOI: 10.1097/JOM.0000000000001215.

6. Cullen MR. The worker with multiple chemical sensitivities: an overview. Occup Med 1987;2:655-61.

7. Fernández MT, Álvarez JC. Anestesia con sevoflurano para cirugía de senos paranasales en un paciente con sensibilidad química múltiple. Rev Esp Anestesiol Reanim. 2018;65(1):49-52. DOI: 10.1016/j.redar.2017.03.018.

8. Lorenzo López M , Hernández Martín D, Ortega Ladrón de Cegama E, Aldecoa Álvarez-Santullano C. Cesárea electiva en una paciente con síndrome de sensibilidad química múltiple. Rev Esp de Anestesiol Reanim. 2014;61(2):61-120. DOI: 10.1016/j.redar.2013.01.005.

9. Fisher M, Rose M. Anaesthesia for patients with idiopathic environmental intolerance and chronic fatigue syndrome. $\mathrm{Br} J$ Anaesth. 2008;101(4):486-91. DOI: 10.1093/bja/aen242.

10. Bustamante R. Anafilaxia a los bloqueadores neuromusculares. Rev Chil Anest. 2011;40(4):316-34. 